\title{
An Improved ARP Protocol for Enhanced Lifetime in Wireless AD Hoc Networks
}

\author{
Qian Zhao ${ }^{1,2}$, Wen $\mathrm{Hu}^{1}$ and Guangsheng Feng ${ }^{2}$ \\ ${ }^{1}$ School of Computer and Information Engineering, Harbin University of Commerce \\ Harbin, 150028, China \\ zhaoqian@hrbeu.edu.cn,huwen@hrbcu.edu.cn \\ ${ }^{2}$ College of computer science and technology, Harbin Engineering University \\ Harbin, 150001, China \\ fengguangsheng@hrbeu.edu.cn
}

\begin{abstract}
We focus on the problem how to achieve network lifetime extension without causing serious deteriorated on network performance. A cooperative E-ARP protocol for extending network lifetime in wireless AD Hoc networks is proposed in our paper. By means of piggyback, energy information exchanges among neighbors in our scheme, which makes a little modification to traditional ARP protocol. E-ARP protocol can carry energy information of wireless nodes, which decreased the risk of communication resource competition between $A R P$ information and exchanging energy information. It also allows the node with sufficient energy to help multiple nodes at one time, thus the extra communication workload can be decreased. In evaluations chapter, the simulation results show that E-ARP protocol can achieve an almost similar performance on lifetime extension with the known method CTCA but better network performance.
\end{abstract}

Keywords: network lifetime; energy; communication workload; topology control

\section{Introduction}

The proliferation of wireless networks based applications requires wireless nodes to live as long as possible. However, current technologies of battery capacity equipped to wireless nodes are hard to improve wireless node's lifetime significantly ${ }^{[1-3]}$. Under this situation, lots of researchers have focused their studies on extension of the network lifetime from the aspect of using energy efficiency.

One scheme to extend the network life by adding redundant nodes in the network is proposed in [3]. This kind of scheme may extend the lifetime significantly but ultimately not a cost-effective approach. Recently, conserving energy and extending a network's lifetime by means of topology control has been increasingly attracting researchers' attentions, in which each node adjusts the power at which it makes its transmissions to reduce the energy consumption to only what is needed to ensure topological goals such as connectivity or coverage. Examples of topology control algorithms include DRNG [4], DLSS [4], STC[5], RATC [6] and CTCA [7].

In most traditional algorithms, the topology of the network is determined at the very beginning of network running where the only consideration for each node is to reduce its transmission power while keeping the graph connected [8-10]. only the algorithm CTCA is a dynamic and distributed scheme for adaptive cooperative topology control in consideration of that node transmission radius may result in an unbalanced energy consumption, leading to 
some nodes exhausting their energy far sooner than some others. So far, most of the existing research works mainly focus on incentive issues in reducing the transmit power for energy conservation by means of topology control. It is also facing a series of heavy traffic workload generated when the topology information exchange among nodes, which may certainly increase the end to end delay and cause packet dropped dramatically.

Unlike the conventional research works, we consider how to extend the network lifetime by means of cooperative and dynamic topology control without impacting heavy workload on it and without causing a mass of packets lost. Both the workload and resource competition is considered in our scheme. One major technical challenge of designing such dynamic scheme is to reducing exchanging messages. This challenge makes our work fundamentally different from any existing solutions. First, different from existing works in which all node broadcasts its energy message independently and it will cause serious communication resource competition as far as to huge packets loss. Secondly, in existing works, one network node only considers how to help its up-link nodes regardless of the down-link notes, which will cause serious network turbulence even significant performance deterioration. The contributions of this work are summarized as follows:

We introduce a novel method to traditional ARP protocol such that it can carry on extra energy exchange messages among neighbors, which is named as E-ARP protocol. Specifically, E-ARP protocol broadcast its energy message by means of piggyback, which will reduce the communication workload and decrease the competition risk among neighbors.

We design a distributed algorithm to evaluate whether one node should help its neighbors to extend lifetime or not. Furthermore, when one node makes sure that it can help one of its neighbors, our algorithm can find out multiple neighbors as many as possible this node can help together.

We evaluate the effectiveness and efficiency of E-ARP by implementing it in a simulation experiment. From the experiment, we demonstrate that our scheme can improve the network utility and decrease loss rate effectively. More important, it also shows that our E-ARP scheme could successfully extend network lifetime to almost the same extent with the existing methods, but without heavy extra communication workload.

The remainder of this paper is organized as follows. In Section II, we introduce notations and sketch for E-ARP, which is useful to understand our work and the E-ARP processes. In Section III, the detail of our scheme is introduced, which design a distributed algorithm to achieve the extension of network lifetime. We evaluate the performance of E-ARP via experiment in Section IV, which is followed by the conclusion and future work.

\section{Notations and sketch for E-ARP Scheme}

\subsection{Description and Hypothesizes for E-ARP Protocol}

In wireless networks, whether the route protocol is AODV, DSR, OLSR or others, the ARP protocol works invoked by two mechanisms, one is invoked by packet arrival from up layer and the other is invoked by timer. In former mechanism, one arrival packet cannot attain a valid physical address from ARP cache, and in later mechanism, the process with ARP protocol will broadcast ARP request packet periodically based on its timer. Our main purpose is to use ARP protocol to exchanging energy information by means of piggyback.

Given a wireless network, let graph $G(t)=(N, E(t))$ represent its topology at time $t$, where $N$ is node set, $E(t)$ is edge set, $N_{i} \in N$ represents a node within the network, and $\left(N_{i}, N_{j}\right) \in E(t)$ represents the fact that $N_{j}$ is within $N_{i}$ 's communication radius and can hear 
from $N_{i}$ directly. Before discussing the proposed scheme, there are two hypothesizes. Notations and definitions used in this paper are as Table 1 shown.

Table 1. Notations and Definitions used in this Paper

\begin{tabular}{|c|c|}
\hline Notation & Definition \\
\hline$N$ & The set of nodes within the network $N=\left\{N_{1}, N_{1}, \cdots, N_{n}\right\}$ \\
\hline$E(t)$ & $\begin{array}{l}\text { The set of edges, if }\left(N_{i}, N_{j}\right) \in E(t) \text {, it means that } N_{j} \text { is within } N_{i} \text { 's communication } \\
\text { radius }\end{array}$ \\
\hline$G(t)$ & $G(t)=(N, E(t))$ represent its topology at time $t$ \\
\hline$N_{i}$ & The node whose numerical order is $i \in\{1,2, \cdots, n\}$ \\
\hline$p\left(N_{i}, N_{j}\right)$ & The minimum transmit power from $N_{i}$ to $N_{j}$ and assumes $p\left(N_{i}, N_{j}\right)=p\left(N_{j}, N_{i}\right)$ \\
\hline$p_{i}(t)$ & $N_{i}$ 's transmit power at time $t$ \\
\hline$H_{i}(t)$ & $N_{i}$ 's down-link node set that $H_{i}(t)=\left\{N_{j} \mid\left(N_{i}, N_{j}\right) \in E(t)\right\}$ \\
\hline$I_{i}(t)$ & $N_{i}$ 's up-link node set that $I_{i}(t)=\left\{N_{j} \mid\left(N_{j}, N_{i}\right) \in E(t)\right\}$ \\
\hline$W_{i}(t)$ & $N_{i}$ 's remaining energy at time $t$ \\
\hline$Z_{i}(t)$ & $N_{i}$ 's remaining lifetime with power $p_{i}(t)$ at time $t$, and $Z_{i}(t)=W_{i}(t) / p_{i}(t)$ \\
\hline$Z_{i}^{\prime}\left(t, N_{j}\right)$ & $N_{i}$ 's potential remaining lifetime if $N_{i}$ 's transmit power is $p\left(N_{i}, N_{j}\right)$ \\
\hline$B_{i}$ & $\begin{array}{l}\text { The available power set of } N_{i} \text {, in which there are } K_{i} \text { items, } \\
\text { and } B_{i}=\left\{B_{i}\left(k_{i}\right) \mid K_{i}=0,1, \cdots, K_{i}-1\right\}=\left\{\left(N_{j}, p\left(N_{i}, N_{j}\right)\right) \mid N_{j} \in H_{i}(t) \wedge \text { order by } p\left(N_{i}, N_{j}\right)\right\}\end{array}$ \\
\hline$B_{i}(s) \rightarrow N$ & The node whose numerical order is $\sin B_{i}$ \\
\hline$B_{i}(s) \rightarrow P$ & The power whose numerical order is $s$ in $B_{i}$ \\
\hline$k_{i}$ & The numerical order of $N_{i}$ 's deciding node in $B_{i}$ \\
\hline$c_{i}\left(N_{j}\right)$ & $\begin{array}{l}\text { If } N_{j} \text { is in some item of } B_{i} \text {, the function returns } N_{j} \text { 's numerical order in } B_{i} \text {, else } \\
\text { return integer of }-1\end{array}$ \\
\hline$N_{d_{i}}$ & $N_{i}$ 's deciding node \\
\hline$m_{i}(i)$ & $\begin{array}{l}\text { the numerical order in } N \text { of } N_{i} \text { 's deciding node, which can be presented as } \\
N_{m(i)}\end{array}$ \\
\hline$R_{i}$ & The set defined as $R_{i}::=\{(N, D, \Delta) \mid$ order by $\Delta\}$, in which there are $r_{i}$ elements. \\
\hline$R_{i}(u) \rightarrow N$ & The node can be helped by $N_{i}$, whose numerical order is $u$ in $R_{i}$ \\
\hline$R_{i}(u) \rightarrow D$ & The deciding node of $R_{i}(u) \rightarrow N$, whose numerical order is $u$ in $R_{i}$ \\
\hline$R_{i}(u) \rightarrow \Delta$ & The difference of remaining lifetime between $N_{i}$ and $R_{i}(u) \rightarrow N$ \\
\hline$R_{i}(u) \rightarrow P^{\prime}$ & $\begin{array}{l}\text { The potential power of node } R_{i}(u) \rightarrow N \text {, means that if } N_{i} \text { provides help, node } \\
R_{i}(u) \rightarrow N \text { can decrease its power to a lower level, i.e., } R_{i}(u) \rightarrow P^{\prime}\end{array}$ \\
\hline$R_{i} \rightarrow \Delta$ & $\begin{array}{l}\text { The difference of remaining lifetime between } N_{i} \text { and its neighbor, if we do not } \\
\text { refer to a specific neighbor }\end{array}$ \\
\hline$\gamma_{i}(u)$ & the node numerical order in $N$ of node $R_{i}(u) \rightarrow N$ \\
\hline$\gamma_{i}^{\prime}\left(N_{i}\right)$ & The element numerical order in $R_{i}$, if $N_{i} \notin R_{i}$, return integer of -1 \\
\hline$S_{i}$ & The set defined as two tuple $S_{i}::=\{(Z, N) \mid$ order by $Z\}$ \\
\hline$S_{i}(v) \rightarrow N$ & The node can help $N_{i}$ extend its lifetime, whose numerical order is $v$ in $S_{i}$ \\
\hline$S_{i}(v) \rightarrow Z$ & The remaining lifetime of node $S_{i} \rightarrow N$, whose numerical order is $v$ in $S_{i}$ \\
\hline$S_{i} \rightarrow Z$ & $\begin{array}{l}\text { The remaining lifetime between } N_{i} \text { and its neighbor, if we do not refer to a } \\
\text { specific neighbor }\end{array}$ \\
\hline
\end{tabular}


Hypothesis 1. Suppose $N_{i}$ 's transmit power at time $\mathrm{t}$ is $p_{i}(t)$ and its communication radius can cover node $N_{j}$. If $N_{j}$ 's transmit power $p_{j}(t)$ is not less than $p_{i}(t), N_{j}$ 's communication radius must be able to cover $N_{i}$.

Hypothesis 2. If $N_{i}$ 's transmit power is $p_{i}(t)$ and its communication radius covers $N_{j}$. If $N_{j}$ 's receiving power can measured, the $N_{i}$ 's minimal transmit power can deduced.

\subsection{States transfer in E-ARP Protocol}

In order to undertake energy exchange among neighborhood nodes, an E-ARP protocol is proposed on the basis of traditional ARP, which includes two stages: initialization and power adjustment stage. In power adjustment stage, traditional ARP is still undertaking the physical address query and detection. In addition, E_DETECT, E_PK, POW_AD are newly added states for energy detection and power adjustment. Figure 1 shows the E-ARP states transfer diagram, in which the blue dotted line and connected states are newly added or modified. For each $N_{i} \in N$, the states transfer process is as Figure 1 shown.

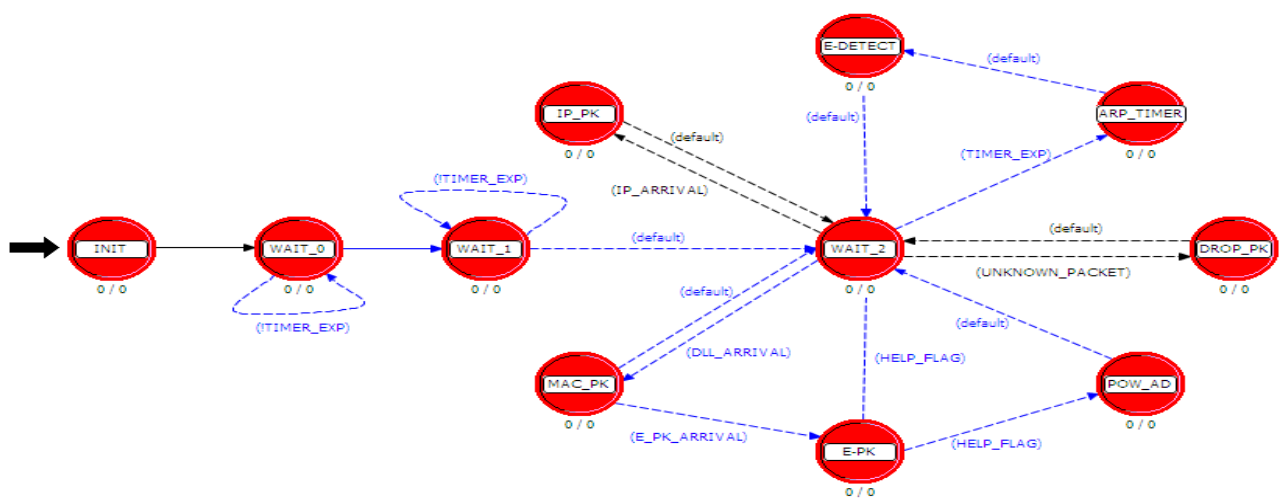

Figure 1. E-ARP State Transfer Diagram

Initialization stage is included of INIT, WAIT_0 and WAIT_1 states, in which all nodes will construct themselves up-link node set $I_{i}(t)$, down-link node set $H_{i}(t)$, and available power set $B_{i}(i=1,2, \cdots, n)$, where $I_{i}(t)=\left\{N_{j} \mid\left(N_{j}, N_{i}\right) \in E(t)\right\}, H_{i}(t)=\left\{N_{j} \mid\left(N_{i}, N_{j}\right) \in E(t)\right\}$, and $B_{i}=\left\{B_{i}(s) \mid s=0,1, \cdots, K_{i}-1\right\}=\left\{\left(N_{j}, p\left(N_{i}, N_{j}\right)\right) \mid N_{j} \in H_{i}(t) \wedge\right.$ order by $\left.p\left(N_{i}, N_{j}\right)\right\}$. When $B_{i}$ constructed successfully, it will remain unchanged in the whole runtime. In state WAIT_1, network $G(t)$ will be optimized to a minimum connected graph based on the algorithm DLSS.

Power adjustment stage includes WAIT_2, MAC_PK, IP_PK, DROP_PK, E_PK, POW_AD, EARP_TIMER and E_DETECT states. In this stage, packet arrival, timer expiration, help flag are three kinds of events that invoke the process from one state transferring to another. The state transferring is presented in section III detailedly.

\section{Process and States Transferring in E-ARP}

\subsection{States in initialization Stage}

INIT state. When the network starts running, it step into INIT state and initialize the EARP protocol, which includes storage allocation, synchronization with above or below layer such as transport layer or mac layer, and so on. Each node $N_{i}$ exchanges energy information 
$\left(N_{i}, p_{\max }, W_{i}(t)\right)$ with its neighbors for constructing up-link set and down-link set, where $p_{\max }$ is the available max power and $W_{i}(t)$ is remaining energy of $N_{i}$. Any node in this state will broadcast its remaining energy to neighbors at the maximum power $p_{\max }$. after initialization finished, the process will transfer to WAIT_0 state automatically without any event invoking.

WAIT_0 state. In this state, the main task for each node is to collect neighbor's information, including neighbor ID, remaining energy. In this state, any other format packet except for E-ARP will not be accepted in this state. On the basis of collection, $N_{i}$ 's up-link set $I_{i}(t)$ can be constructed. Because each neighbor in INIT state will broadcast its energy information with the maximum power $p_{\max }$, the minimum power level from $N_{i}$ to its neighbor $N_{j} \in I_{i}(t)$ can be inferred according to hypothesis 1 and hypothesis 2., i.e., $p\left(N_{i}, N_{j}\right)$. Thus, the down-link set $H_{i}(t)$ can also be constructed correspondingly, in which there are $K_{i}$ elements. In $N_{i}$ 's available power set defined as $B_{i}$, its items are sorted with ascending order of $p\left(N_{i}, N_{j}\right)$. Thus, it is obvious that $N_{i}$ 's transmit power is decided by the node in last item of $B_{i}$, i.e., $N_{m_{i}\left(K_{i}\right)}=B_{i}\left(K_{i}\right) \rightarrow N$, named as "deciding node" of $N_{i}$, where $B_{i}\left(K_{i}\right)$ denotes the $K_{i}$ th item, and $N_{m_{i}\left(K_{i}\right)}, B_{i}\left(K_{i}\right) \rightarrow N$ are equally denote the node in item $B_{i}\left(K_{i}\right)$. Thus, $p\left(N_{i}, N_{m_{i}\left(K_{i}\right)}\right)$ and $B_{i}\left(K_{i}\right) \rightarrow P$ are equally denote the transmit power value of $B_{i}\left(K_{i}\right)$. The process will stay in this state to continue collecting information and constructing its corresponding sets $I_{i}(t), H_{i}(t)$ and $B_{i}$ until the timer expired, when the process transfer WAIT_1 state automatically. Here, the timer is set properly and used to wait all the broadcasted E-ARP packets.

WAIT_1 state. On the basis of $I_{i}(t), H_{i}(t)$ and $B_{i}$ constructed in WAIT_0, run DLSS algorithm to determine node $N_{i}$ 's transmit power $p_{i}(t)$ with the constraint of minimum connected graphic of network $G(t)$. Although any format packet will not be accepted in this state, it is still necessary to set up a timer to synchronize with other nodes. When the timer is expired and $k_{i}>1$, broadcast its energy information $\left(W_{i}(t), p_{i}(t), N_{m_{i}\left(k_{i}\right)}, B_{i}\left(k_{i}-1\right) \rightarrow P\right)$ to its neighbors, where $N_{m_{i}\left(k_{i}\right)}$ is node $N_{i}$ 's deciding node, i.e., $N_{m_{i}\left(k_{i}\right)}=B_{i}\left(k_{i}\right) \rightarrow N$, and $B_{i}\left(k_{i}-1\right) \rightarrow P$ is $N_{i}$ 's potential transmit power. In other words, if one node $N_{j} \in H_{i}$ increases its power to cover $N_{m_{i}\left(k_{i}\right)}, N_{i}$ can decrease its power to a lower level $B_{i}\left(k_{i}-1\right) \rightarrow P$, and all the packets from $N_{i}$ to $N_{m_{i}\left(k_{i}\right)}$ can through relaying node $N_{j}$. Notice that $N_{i}$ 's deciding node is $N_{m_{i}\left(k_{i}\right)}$ instead of $N_{m_{i}\left(K_{i}\right)}$, which is because each node's transmit power is decreased to just enough to maintain $G(t)$ as a minimum connected graphic. Therefore, the set $I_{i}(t)$ and $H_{i}(t)$ is changed with the transmit power. On the contrary, set $B_{i}$ remains unchanged in the whole runtime. At the end, the process is transferred to WAIT_2 state automatically.

\subsection{States in Power Adjustment Stage}

There are several events being happened in WAIT_2 state, which are included of timer being expired, E-ARP packets being arrived and IPpackets being arrived. Different events will invoke this process to different process states, such as Error! Reference source not found. shown.

Event condition: IP_ARRIVAL. If this condition is turned true which means an IP packet arrives from above layer, the arrived IP packet will be delivered to IP_PK state for further processing. 
Event condition: UNKNOWN_PACKET. If this condition is turned true, a packet with unknown format arrives. Under this case, it will be transferred to DROP_PK state and drop it directly.

No matter it is an IP_ARRIVAL event or an UNKNOWN_PACKET event, the way of handling on them is nothing different with the traditional ARP protocol. How to assist neighbors to extend their lifetime or how to accept the help from neighbors are our key consideration in this paper.

Event condition: TIMER_EXP. If this condition is turned true, it is a new round for updating ARP cache and the process will transfer to ARP_TIMER state. Traditionally, the ARP request message will be broadcasted to its neighbors. Thus, the energy information can be carried on this message by means of piggyback. In order to achieve this, the data fields should be added to the ARP format. Although the new packet size is a little larger than before, it is worth to share broadcast channel resource at a cost of a little modification. Therefore, the energy message will be broadcasted in E-DETECT state.

In our proposed protocol, sets $R_{i}$ and $S_{i}$ are critical important. $R_{i}$ defined as $R_{i}::=\{(N, D, \Delta) \mid$ order by $\Delta\}$, includes some information of neighbors in $I_{i}$, whose lifetime can be extended with assistance from $N_{i} . R_{i}(u)$ denotes the uth item in $R_{i}, R_{i}(u) \rightarrow N$ denotes the node included in $R_{i}(u), R_{i}(u) \rightarrow D$ denotes the deciding node of $R_{i}(u) \rightarrow N$ and $R_{i}(u) \rightarrow \Delta$ denotes the difference of remaining lifetime between $N_{i}$ and $R_{i}(u) \rightarrow N . S_{i}$ defined as $S_{i}::=\{(Z, N) \mid$ order by $Z\}$, includes some information of neighbors in $H_{i}$, which can provide help to extend $N_{i}$ 's lifetime. $S_{i}(u) \rightarrow N$ denotes the node included in the uth item, i.e., $S_{i}(u)$, and $S_{i}(u) \rightarrow Z$ denotes $S_{i}(u) \rightarrow N$ 's remaining time.

Event Condition: DLL_ARRIVAL and E_PK_ARRI-VAL. If both conditions are turned true in due order, this process will transfer from WAIT_2 to MAC_PK and then to E_PK. If some neighbor's energy information is carried by the arrived packet, set $R_{i}$ and $S_{i}$ will be constructed as shown in Figure 2 and Figure 3.

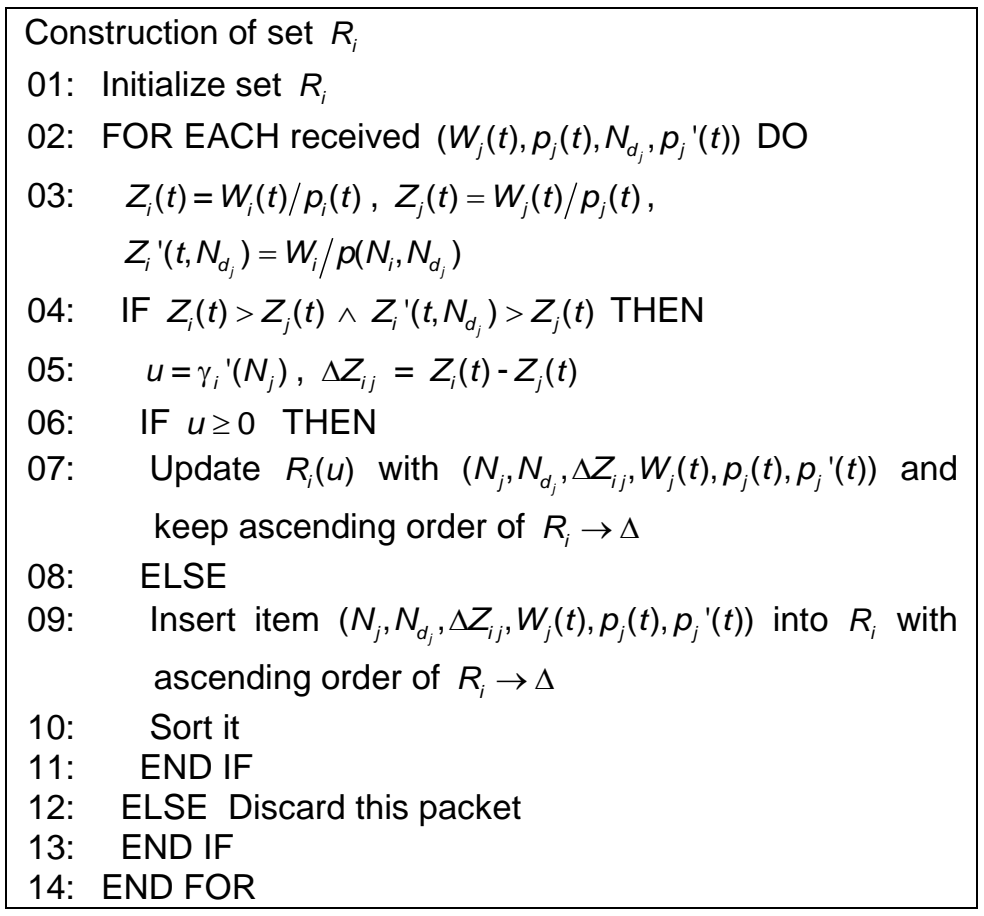


15: Get the set of $R_{i}$ 's size: $r_{\text {size }}$

16: Send $\left(W_{i}(t), p_{i}(t), C A N_{-} H E L P\right)$ to node $R_{i}\left(r_{\text {size }}-1\right) \rightarrow N$

Figure 2. Construction of Set $R_{i}$

Line 02-14 in Figure 2: for any $N_{j} \in N$, its remaining time $Z_{j}(t)$ will be calculated and compared with the current node $N_{i}$ 's remaining time $Z_{i}(t) . N_{d_{j}}$ is $N_{j}$ 's deciding node and $p\left(N_{i}, N_{d_{j}}\right)$ is potential transmit power from $N_{i}$ to $N_{d_{j}}$. In other words, if $N_{i}$ increases its power to $p\left(N_{i}, N_{d_{j}}\right)$ that $N_{d_{j}}$ can be covered, $N_{i}$ 's potential remaining lifetime $Z_{i}{ }^{\prime}\left(t, N_{d_{j}}\right)$ calculated in line 03 is still longer than $N_{j}$ 's. Under this case, $N_{i}$ 's power can increase to $p\left(N_{i}, N_{d_{j}}\right)$ in next state POW_AD safely. Otherwise, $N_{i}$ 's power remains unchanged in this adjustment round. All nodes which $N_{i}$ can help will be inserted into $R_{i}$ with its energy information, and further sort them with ascending order of $R_{i} \rightarrow \Delta$.

Line 15-16 in Figure 2: get the node $R_{i}\left(r_{\text {size }}-1\right) \rightarrow N$ in last item of $R_{i}$, which has the minimum lifetime in $I_{i}$, and send the $N_{i}$ 's remaining energy, transmit power and "CAN_HELP" signal to it. Because $R_{i}\left(r_{\text {size }}-1\right) \rightarrow N$ is in $I_{i}$, the sending message must be send in unicast way by means of route protocol. The computation complexity of this process is decided by loop 02-14 in Figure 2 and set operation 09 in Figure 2, which is $O(n \log n)$.

If an E-ARP packet with "arpopcode" value CAN_HELP from node $N_{j}$, the current node $N_{i}$ will update its set $S_{i}$ as shown in Figure 3. Its computation complexity is decided by loop 02-07 in Figure 3 and set insertion with order 05 in Figure 3, so its computation complexity is $\mathrm{O}(n \log n)$.

If an E-ARP packet with CAN_HELP from $N_{j}, N_{i}$ will update $S_{i}$. If $Z_{j}(t)>Z_{i}(t)$, insert $\left(Z_{j}(t), N_{j}\right)$ into $S_{i}$ with ascending order of $S_{i} \rightarrow \Delta$. Its computation complexity is decided by set insertion, which is $\mathrm{O}(n \log n)$.

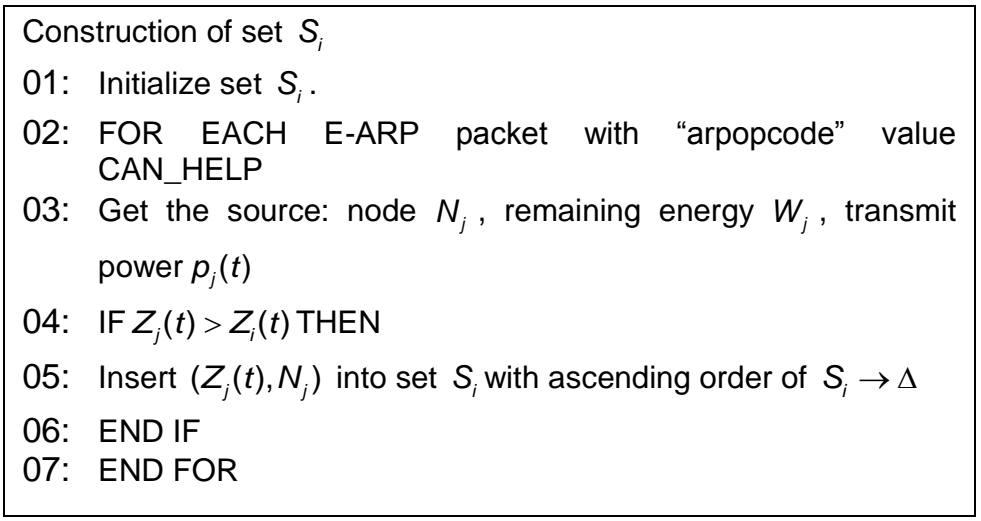

Figure 3. Construction of Set $S_{i}$

There will be four cases for $R_{i} S_{i}$. Case 1: both $R_{i}$ and $S_{i}$ are empty; case 2: neither $R_{i}$ nor $S_{i}$ is empty; case 3: $S_{i}$ is not empty but $R_{i}$ empty; and case 4: $R_{i}$ is not empty but $S_{i}$ empty. 
If an E-ARP packet with CAN_HELP from $N_{j}, N_{i}$ will update $S_{i}$. If $Z_{j}(t)>Z_{i}(t)$, insert $\left(Z_{j}(t), N_{j}\right)$ into $S_{i}$ with ascending order of $S_{i} \rightarrow \Delta$. Its computation complexity is decided by set insertion, which is $\mathrm{O}(n \log n)$.

There will be four cases for $R_{i} S_{i}$. Case 1: both $R_{i}$ and $S_{i}$ are empty; case 2: neither $R_{i}$ nor $S_{i}$ is empty; case 3: $S_{i}$ is not empty but $R_{i}$ empty; and case 4: $R_{i}$ is not empty but $S_{i}$ empty.

Case 1 means neither $N_{i}$ can help nodes in $I_{i}$, nor be helped by nodes in $H_{i}$. Under this case, the whole lifetime of the network is decided by $N_{i}$. If there exist multiple nodes whose $R_{j}$ and $S_{j}$ are empty, the lifetime of network is $\min \left\{Z_{j}\right\}$. Case 2 means $N_{i}$ can help some node in $R_{i}$ by way of increasing its power. On the contrary, $N_{i}$ can reduce transmit power to extend its lifetime with other node's help in $S_{i}$. Under this case, $N_{i}$ should not change transmit power until case 4 appears. On the contrary, no matter how $N_{i}$ 's transmit power is changed; there will be a serious turbulence, which will result in a poor network performance. Case 3 means $N_{i}$ cannot help any node in $I_{i}$. Thus, there must be existed a node $N_{j} \in H_{i}$, so that $N_{i}$ is included in one item of $N_{j}$ 's $R_{j}$ set. Under this case, $N_{i}$ should maintain its current power, and wait other node in $S_{i}$ to increase communication radius to cover $N_{i}$ 's deciding node. On the contrary, if $N_{i}$ decreases transmit power to a lower level before received a "HAS_HELP" signal, $G(t)$ will be disconnected possibly. In case 1-3, HELP_FLAG is false, and the process will return to WAIT_2 state. Only in case 4, this condition is turned true and this process is invoked to POW_AD.

Event condition: HELP_FLAG. When this condition is true, the process will transfer to POW_AD state. Suppose that there are $r_{i}$ elements in set $R_{i}$, in which the numerical order of elements is from 0 to $r_{i}-1$. Node $N_{i}$ will lookup set $R_{i}$ and find out more short-lifetime nodes to help as much as possible. The pseudocode of transmit power adjustment is shown in Figure 4, where $c_{i}\left(R_{i}\left(e_{n}\right) \rightarrow D\right)$ returns node $R_{i}\left(e_{n}\right) \rightarrow D$ 's numerical order in $B_{i}$ if $c_{i}\left(R_{i}\left(e_{n}\right) \rightarrow D\right)$ is in some item of, else returns -1. $\gamma_{i}\left(e_{n}\right)$ returns node $R_{i}\left(e_{n}\right) \rightarrow N$ numerical order in $\mathrm{N}$ of node.

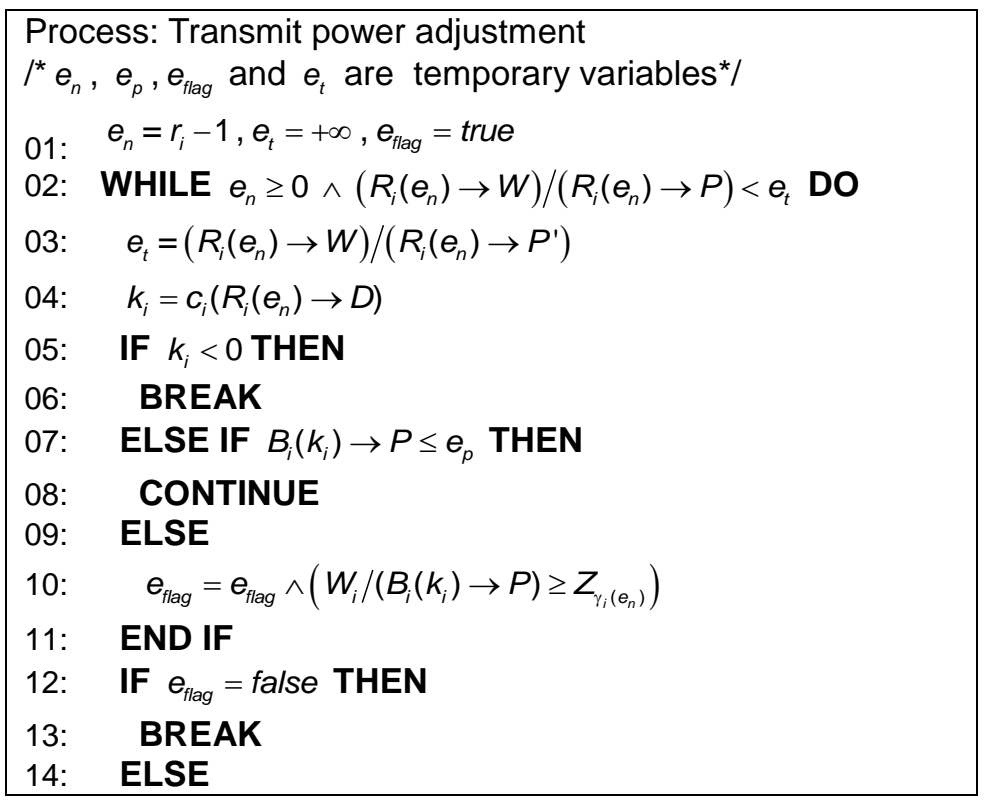




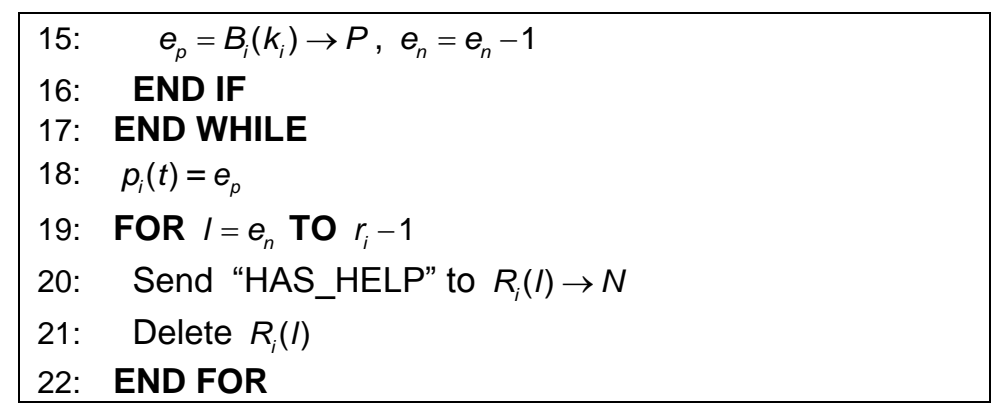

Figure 4. Increasing Power to Help Multiple Neighbors

Line $02-17$ in Figure 4 get the nodes as many as possible that node $N_{i}$ can provide help. Conditions in 02 mean that if the lifetime of the node that is going to be examined in $R_{i}$ is lessen than all potential lifetimes of the nodes that having been examined, this loop continues. Otherwise, it is meaningless to extend its lifetime, because this node being examined is not the one with minimum lifetime and cannot decide the network lifetime.

Line 07-08 in Figure $4 B_{i}\left(k_{i}\right) \rightarrow P \leq e_{p}$ means that $N_{i}$ 's tentative power $e_{p}$ can cover the deciding node $R_{i}\left(e_{n}\right) \rightarrow D$ of $R_{i}\left(e_{n}\right) \rightarrow N$. Thus, the transmit power of $R_{i}\left(e_{n}\right) \rightarrow N$ is allowable to decrease to a lower level. The assignment statement in line 10 is used to check whether the node examined can be helped or not.

Line 18-22 in Figure 4 node $N_{i}$ 'transmit power has been determined and the set $R_{i}$ is update, in which all nodes with $N_{i}$ 's help are deleted. The computation complexity is decide by loop 02-17 in Figure.4or by loop 19-22 in Figure.4, and it is obvious that its computation complexity is $\mathrm{O}(n)$.

\section{Evaluations}

In this section, we evaluate the effectiveness and efficiency of the proposed E-ARP from following aspects: 1) Setup of our experiments; 2) Evaluation of the effectiveness in network lifetime extension; 3) Evaluation of the network performance.

The experiment setup is the same with [7]. The energy model used is the same with that used in [11]. Our simulation is conducted for a square $10 \mathrm{kmxl0km}$ region within which 200 nodes are placed in random locations. Each node is equipped with $40 \mathrm{~kJ}$ of energy and has a maximum transmission power $p_{\max }$, which corresponds to a transmission radius of $20 \%$ of the width of the square region.

Figure 5 shows the network lifetime achieved by E-ARP scheme and CTCA scheme, in which $\mathrm{x}$-axis indicates the number of rounds that has passed while $\mathrm{y}$-axis indicates the percentage of graphs that are still connected. Compared with CTCA, our scheme is a little poor in first 17 rounds, but after 17 rounds, it is a little better than CTCA. Overall, our scheme is achieved almost the same performance. However, our scheme is better than CTCA in respect of data dropped, delay and retransmission times, especially in network throughput, our scheme is superior to CTCA obviously, as shown in Figure 6, Figure 7, Figure 8 and Figure 9. Thus, our E-ARP scheme has achieved an almost similar performance on lifetime extension with CTCA. More important, our scheme has a better network performance.

Because our scheme employs modified ARP packet to exchange energy message by means of piggyback, this kind of information will be broadcasted with ARP request packet and share the communication channel. Thus, the dropped data caused by competing communication 
resource will be decreased, just as shown in Figure 6. In Figure 7, our scheme is superior to CTCA in overall, and only in the beginning of simulation, it is a little poor resulted by controlling help process as shown in Figure 4. Correspondingly, the performance on average retransmission times is better than CTCA, as shown in Figure 8. In Figure 9 it is observed that our scheme has achieved an outstanding performance on network throughput because of less fewer data dropped, delay and retransmissions.

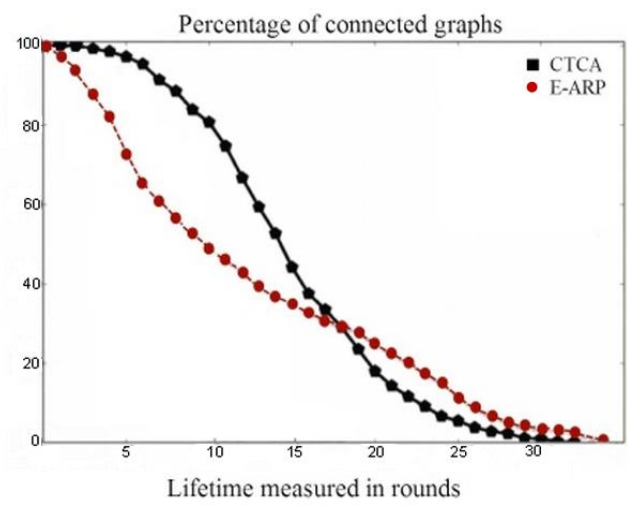

Figure 5. Effectiveness in Network Lifetime Extension

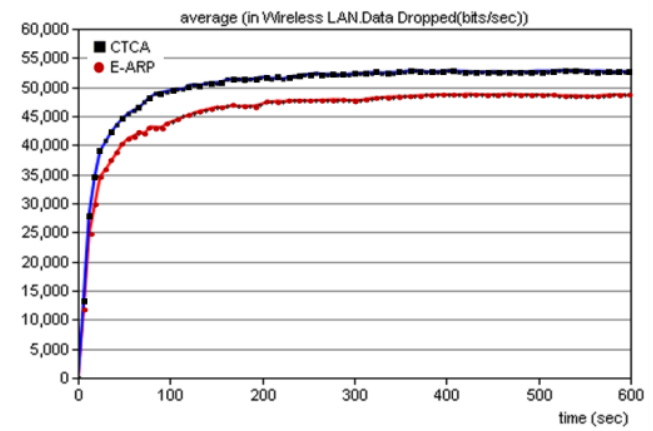

Figure 6. Average Data Dropped between CTCA and E-ARP

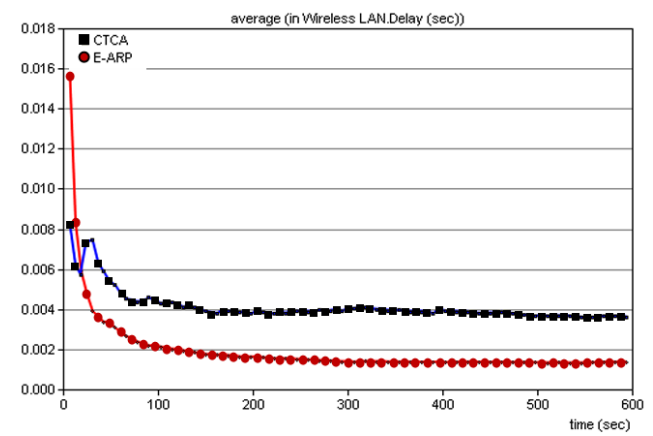

Figure 7. Average Delay Between CTCA and E-ARP 


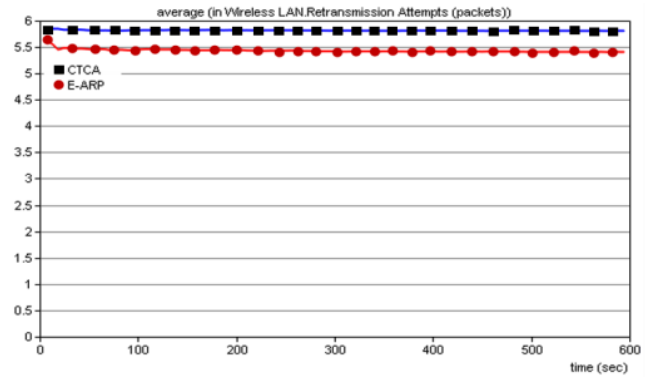

Figure 8. Average Retransmission Attempts between CTCA and E-ARP

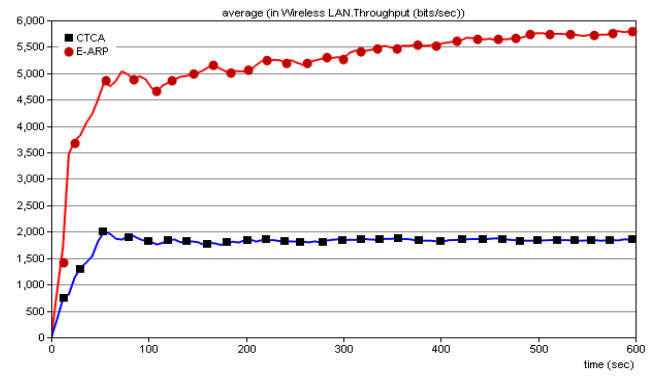

Figure 9. Average Throughput between CTCA and E-ARP

\section{Conclusion}

This paper focuses on the problem how to achieve network lifetime extension without causing serious deteriorated on network performance. We propose an E-ARP scheme based on traditional ARP and a little modification on it. E-ARP scheme enables energy message exchange among neighbors by means of piggyback, which decrease the risk of communication resource competition. Furthermore, our scheme allows the node with sufficient energy to help more than one node as many as possible at one time, thus the extra communication workload can be decreased also. We conduct a simulation to evaluate the proposed scheme and demonstrate the effectiveness compared with CTCA.

\section{Acknowledgments}

This work was supported in part by the Research Fund for the Doctoral Program of Higher Education of China under Grant 20122304130002, the Natural Science Foundation of Heilongjiang Province under Grant ZD 201102 and F201037, the Fundamental Research Fund for the Central Universities under Grant HEUCFZ1213 and HEUCF100601, Science and Technology Research Projects of Department of Education in Heilongjiang Provincial under Grant 12521135, and Postdoctoral Science Foundation of Heilongjiang Province under Grant LBH-210204.

\section{References}

[1] P. Mahadevan, P. Sharma, S. Banerjee and P. Ranganathan, "Energy aware network operations", Proceedings of the INFOCOM Workshops, (2009), pp. 1-6.

[2] G. Anastasi, M. Conti, M. Di Francesco and A. Passarella, "Energy conservation in wireless sensor networks: A survey", Ad Hoc Networks, vol. 7, no. 3, (2009), pp. 537-568.

[3] Y. T. Hou, Y. Shi, H. D. Sherali and S. F. Midkiff, "On energy provisioning and relay node placement for wireless sensor networks", Wireless Communications, IEEE Transactions, vol. 4, no. 5, (2005), pp. 25792590. 
[4] N. Li and J. C. Hou, "Localized topology control algorithms for heterogeneous wireless networks", IEEE/ACM Transactions on Networking (TON), vol. 13, no. 6, (2005), pp. 1313-1324.

[5] H. Sethu and T. Gerety, "A new distributed topology control algorithm for wireless environments with nonuniform path loss and multipath propagation", Ad Hoc Networks, vol. 8, no. 3, (2010), pp. 280-294.

[6] R. S. Komali, A. B. MacKenzie and P. Mahonen, "On selfishness, local information, and network optimality: A topology control example", Proceedings of 18th International Conference on Computer Communications and Networks, (2009), pp. 1-7.

[7] X. Chu and H. Sethu, "Cooperative topology control with adaptation for improved lifetime in wireless ad hoc networks", Proceedings of 31st International Conference on Computer Communications, (2012), pp. 262-270.

[8] B. Zebbane, M. Chenait and N. Badache, "GTC: a geographical topology control protocol to conserve energy in wireless sensor networks", International Journal of Trust Management in Computing and Communications, vol. 1, no. 3, (2013), pp. 320-340.

[9] S. Zarifzadeh, N. Yazdani and A. Nayyeri, "Energy-efficient topology control in wireless ad hoc networks with selfish nodes", Computer Networks, vol. 56, no. 2, (2012), pp. 902-914.

[10] T. M. Chiwewe and G. P. Hancke, "A distributed topology control technique for low interference and energy efficiency in wireless sensor networks", IEEE Transactions on Industrial Informatics, vol. 8, no. 1, (2012), pp. 11-19.

[11] G. Koltsidas and F.-N. Pavlidou, "A game theoretical approach to clustering of ad-hoc and sensor networks", Telecommunication Systems, vol. 47, no. 1-2, (2011), pp. 81-93.

\section{Authors}

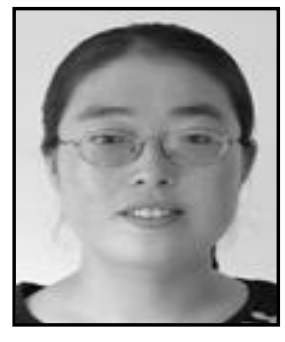

Qian Zhao, received the B.S., M.S. and Ph.D. degrees in computer science from College of Computer Science and Technology, Harbin Engineering University (HEU), China, in 2003, 2006 and 2010 respectively. Currently she is a LECTURER in School of Computer and Information Engineering, Harbin University of Commerce. Her current research interests include software dependability growth, software dependability evaluation and Ad Hoc network.

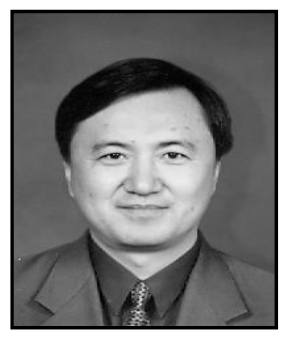

Wen Hu, Computer Society as executive director of Heilongjiang Province, Heilongjiang Province, vice president of e-commerce. He has been engaged in business intelligence, information services, embedded software and other areas of research. In recent years, he completed over 10 research projects. He won provincial and ministerial level scientific and technological progress prize two, third two, published dozens of papers. He is currently Harbin University of Commerce Computer and Information Engineering, a professor. Networked environment, from things he perceived, embedded software research platform configuration.

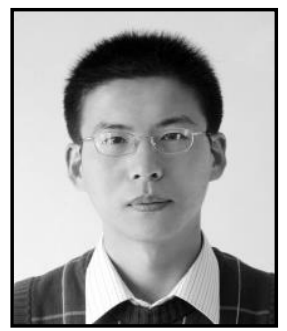

Guangsheng Feng, received B.S., M.S and D.S. degree from Harbin Engineering University in 2003, Harbin Institute of Technology in 2005, and Harbin Engineering University in 2009 respectively. Now, he is engaging in teaching and researching at Cognitive Networks. He is a full stuff at Harbin Engineering University. His research interests involve cross-layer design, information sensing and wireless channel access control. 